\title{
Exact Performance Analysis of Dual-Hop Semi-Blind AF Relaying over Arbitrary Nakagami- $m$ Fading Channels
}

\author{
Minghua Xia, Chengwen Xing, Yik-Chung Wu, and Sonia Aïssa, Senior Member, IEEE
}

\begin{abstract}
Relay transmission is promising for future wireless systems due to its significant cooperative diversity gain. The performance of dual-hop semi-blind amplify-and-forward (AF) relaying systems was extensively investigated, for transmissions over Rayleigh fading channels or Nakagami- $m$ fading channels with integer fading parameter. For the general Nakagami- $m$ fading with arbitrary $m$ values, the exact closed-form system performance analysis is more challenging. In this paper, we explicitly derive the moment generation function (MGF), probability density function (PDF) and moments of the endto-end signal-to-noise ratio (SNR) over arbitrary Nakagami- $m$ fading channels with semi-blind AF relay. With these results, the system performance evaluation in terms of outage probability, average symbol error probability, ergodic capacity and diversity order, is conducted. The analysis developed in this paper applies to any semi-blind AF relaying systems with fixed relay gain, and two major strategies for computing the relay gain are compared in terms of system performance. All analytical results are corroborated by simulation results and they are shown to be efficient tools to evaluate system performance.
\end{abstract}

Index Terms-Amplify-and-forward (AF), dual-hop, Meijer's $G$-function, Nakagami- $m$ fading, performance analysis, semiblind relaying.

\section{INTRODUCTION}

$\mathbf{R}$ ELAY transmission is promising for next-generation wireless systems, owing to its significant cooperative diversity gain [1]. A relay can be exploited to help the source forward signals to the destination if the direct channel from the source to the destination is in deep fading. In general, there are two kinds of relaying schemes, namely, decode-and-forward (DF) and amplify-and-forward (AF). In contrast to DF relay, AF relay does not implement decoding operation and it simply amplifies the received signals prior to transmission; therefore, it is transparent to the modulation and coding schemes of the source.

Manuscript received December 22, 2010; revised April 16, 2011; accepted June 28,2011 . The associate editor coordinating the review of this paper and approving it for publication was A. Gulliver.

M. Xia is with the Division of Physical Sciences and Engineering, King Abdullah University of Science and Technology (KAUST), Thuwal, Saudi Arabia (e-mail: minghua.xia@kaust.edu.sa).

C. Xing is with the School of Information and Electronics, Beijing Institute of Technology, Beijing, China (e-mail: xingchengwen@gmail.com).

Y.-C. Wu is with the Department of Electrical and Electronic Engineering, The University of Hong Kong, Hong Kong (e-mail: ycwu@eee.hku.hk).

S. Aïssa is with INRS-EMT, University of Quebec, Montreal, QC, Canada, and with KAUST, Thuwal, Saudi Arabia (e-mail: sonia.aissa@ieee.org).

The work was supported in part by the HKU Seed Funding Programme, Project No. 200911159084, and by King Abdullah University of Science and Technology.

Digital Object Identifier 10.1109/TWC.2011.080311.102267
In the AF technique, the relay gain aims to invert the firsthop channel while limiting the output power of the relay when the first-hop fading amplitude is low. Generally, there are three methods to determine the relay gain. The first method consists in fixing the relay gain regardless of the fading amplitude of the first-hop channel. Its performance degrades significantly if the channel amplitude varies a lot. The second method exploits full channel state information (CSI). It requires a continuous estimate of the instantaneous first-hop channel amplitude, thus resulting in heavy training overhead. The third method, on the other hand, exploits only statistical CSI of the first-hop channel and it is called the semi-blind relay. Comparing with the fullCSI assisted relay, the semi-blind AF relay is more practical to be deployed, and it only introduces slight performance degradation [2], [3].

The performance of dual-hop semi-blind AF relay has been extensively investigated, especially over Rayleigh fading channels [1], [2], [4]-[7]. However, it is well-known that the Nakagami- $m$ fading is more general and it can model extensive fading scenarios. For performance analysis of dualhop relay under Nakagami- $m$ fading channels, in order to obtain closed-form performance metrics, it is usually assumed that both consecutive hops (source-to-relay and relayto-destination) experience fading with integer $m$ values [3], [8]-[14]. However, the propagation environments where the Nakagami fading parameter takes non-integer values are very common in practice, such as micro-cellular scenarios with strong specular components and land mobile satellite channels [15], [16]. Therefore, accurate performance evaluation of the relaying transmission over arbitrary Nakagami- $m$ fading channels becomes extremely important for practical purpose.

For the scenario with arbitrary $m$ values, the lower bound on average symbol error probability (SEP) of full-CSI assisted relaying transmission is studied in [17], by exploiting the conventional probability density function (PDF) based integration method. In [18], the moment generation function (MGF)-based approach is applied to analyze the average SEP, where the MGF is expressed by the Kampé de Feriét's function. Due to its high complexity, the Kampé de Feriét's function is hard to be further processed and, therefore, the average SEP is obtained by numerical integration [18]. In [19], a comprehensive framework for exact performance analysis over generalized fading channels is presented, including the Nakagaimi- $m$ fading channels with integer $m$ values, while for the Nakagami- $m$ fading scenario with non-integer $m$, 
performance bounds are obtained. To the best of the authors' knowledge, exact and general performance analysis of dualhop relaying over Nakagami- $m$ fading with arbitrary $m$ values remains an open problem. This paper aims at filling this important gap.

Specifically, dual-hop semi-blind AF relay over arbitrary Nakagami- $m$ fading channels is considered in this paper. A general and compact MGF expression for the end-to-end SNR is first derived, by exploiting the generalized Meijer's $G$ function and Fox's $H$-function of two variables. The MGF is further processed and the PDF of the end-to-end SNR is explicitly developed. From the analytical MGF and PDF expressions, four important performance measures, namely, outage probability, average SEP, ergodic capacity and diversity order, are analytically obtained. In addition, the moments of the end-to-end SNR are derived and are further exploited to approximately evaluate the ergodic capacity. All analytical results developed in this paper are corroborated by simulation results, indicating the accuracy of the provided results.

The rest of this paper is organized as follows. Section II describes the system model. The MGF, PDF and moments of the end-to-end SNR are derived in Section III. Four system performance measures, including outage probability, average SEP, ergodic capacity and diversity order, are investigated in Section IV. Numerical results are presented in Section V. Finally, Section VI draws the conclusion.

\section{SySTEM Model}

We consider a dual-hop relaying system with a single AF relay. The source, relay, and destination are equipped with single half-duplex antenna. Both the first hop (source-to-relay) and the second hop (relay-to-destination) experience independent but not necessarily identically distributed Nakagami- $m$ fading with fading shape factors $m_{1}, m_{2} \geq 0.5$, respectively.

The transmission period from the source to the destination is divided into two consecutive phases. During the first transmission phase, the source $S$ transmits signal $x$ with energy $E_{S}$ to the relay $R$. Accordingly, the received signal at the relay is given by

$$
y_{S R}=h_{S R} x+n_{S R},
$$

where $h_{S R}$ denotes the complex channel gain between the source and the relay, and $n_{S R}$ is the additive white Gaussian noise (AWGN) at the relay with zero mean and variance $\sigma_{R}^{2}$.

During the second phase, the source keeps silent while the relay amplifies its received signal $y_{S R}$ and forwards it to the destination $D$. Consequently, the received signal at the destination is

$$
\begin{aligned}
y_{R D} & =\beta h_{R D}\left(h_{S R} x+n_{S R}\right)+n_{R D} \\
& =\beta h_{R D} h_{S R} x+\beta h_{R D} n_{S R}+n_{R D},
\end{aligned}
$$

where $\beta$ stands for the relay gain, $h_{R D}$ denotes the complex channel gain between the relay and the destination, and $n_{R D}$ is the AWGN at the destination with zero mean and variance $\sigma_{D}^{2}$. According to (2), the end-to-end SNR from the source to the destination is given by [2]

$$
\gamma=\frac{\left|\beta h_{R D} h_{S R}\right|^{2} E_{S}}{\left|\beta h_{R D}\right|^{2} \sigma_{R}^{2}+\sigma_{D}^{2}}=\frac{\gamma_{1} \gamma_{2}}{\gamma_{2}+c}
$$

where $\gamma_{1} \triangleq\left|h_{S R}\right|^{2} E_{S} / \sigma_{R}^{2}$ and $\gamma_{2} \triangleq\left|h_{R D}\right|^{2} E_{S} / \sigma_{D}^{2}$ refer to the instantaneous SNRs at the first hop and at the second hop, respectively, and $c$ is a constant for a fixed relay gain $\beta$ :

$$
c \triangleq \frac{E_{S}}{\beta^{2} \sigma_{R}^{2}} .
$$

\section{The MGF, PDF And Moments of THE END-TO-End SNR}

In this section, the exact closed-form MGF, PDF and moments of the end-to-end SNR under arbitrary Nakagami$m$ fading channels are derived.

\section{A. MGF of the End-to-End SNR}

With the assumption of Nakagami- $m$ fading channels, the received SNR $\gamma_{i}$ at the $i^{\text {th }}$ hop, where $i=1,2$, is of Gamma distribution given by [20, Eq. (2.21)]:

$$
f_{\gamma_{i}}\left(\gamma_{i}\right)=\underbrace{\frac{m_{i}^{m_{i}}}{\Gamma\left(m_{i}\right) \bar{\gamma}_{i}^{m_{i}}}}_{\triangleq A_{i}} \gamma_{i}^{m_{i}-1} \exp \left(-\frac{m_{i}}{\bar{\gamma}_{i}} \gamma_{i}\right),
$$

where $\Gamma($.$) is the Gamma function, \bar{\gamma}_{1} \triangleq$ $\mathbb{E}\left\{\left|h_{S R}\right|^{2}\right\} E_{S} / \sigma_{R}^{2}=m_{1}\left(m_{1}+1\right) E_{S} / \sigma_{R}^{2}$, and $\bar{\gamma}_{2} \triangleq \mathbb{E}\left\{\left|h_{R D}\right|^{2}\right\} E_{S} / \sigma_{D}^{2}=m_{2}\left(m_{2}+1\right) E_{S} / \sigma_{D}^{2}$, with $\mathbb{E}\{$.$\} denoting the statistical expectation operator.$

For the semi-blind AF relay transmission, the relay gain is determined by the channel statistics at the first hop. In general, there are two major strategies to calculate the relay gain:

1) Strategy I: The square of relay gain is given by [1]

$$
\beta_{I}^{2}=\frac{E_{S}}{\mathbb{E}\left\{\left|h_{S R}\right|^{2}\right\} E_{S}+\sigma_{R}^{2}}=\frac{E_{S}}{\sigma_{R}^{2}\left(\bar{\gamma}_{1}+1\right)} .
$$

2) Strategy II: The square of relay gain is given by [2]

$$
\beta_{I I}^{2}=\mathbb{E}\left\{\frac{E_{S}}{\left|h_{S R}\right|^{2} E_{S}+\sigma_{R}^{2}}\right\}=\frac{E_{S}}{\sigma_{R}^{2}} \mathbb{E}\left\{\frac{1}{\gamma_{1}+1}\right\} .
$$

Substituting (5) into (7) yields

$$
\begin{aligned}
\beta_{I I}^{2} & =\frac{A_{1} E_{S}}{\sigma_{R}^{2}} \int_{0}^{\infty} \frac{\gamma_{1}^{m_{1}-1}}{\gamma_{1}+1} \exp \left(-\frac{m_{1}}{\bar{\gamma}_{1}} \gamma_{1}\right) \mathrm{d} \gamma_{1} \\
& =\frac{A_{1} E_{S} \Gamma\left(m_{1}\right)}{\sigma_{R}^{2}} \Psi\left(m_{1}, m_{1} ; \frac{m_{1}}{\bar{\gamma}_{1}}\right),
\end{aligned}
$$

where we exploited [21, vol. 1, Eq. (2.3.6.9)] in (8) with $\Psi(a, b ; z)$ being the Tricomi confluent hypergeometric function [21, vol. 5, p. 593].

From (6) and (8), it is clear that once $E_{S}, \sigma_{R}^{2}$ and $m_{1}$ are known, $\beta_{I}^{2}$ and $\beta_{I I}^{2}$ are fixed. Since the end-to-end SNR expression in (3) depends only on the relay gain $\beta$, through $c$ in (4), the following analysis holds for any semi-blind AF relaying systems with fixed relay gain. Therefore, in the following, we do not distinguish the analysis for the two strategies above, and their system performance and comparison will be examined in detail in Section V.

With a fixed relay gain $\beta, c$ in (4) is fixed and, thus, we can calculate the MGF of the end-to-end SNR in (3). This is summarized in the following theorem.

Theorem 1: The MGF of the end-to-end SNR of dual-hop semi-blind AF relaying systems over arbitrary Nakagami- $m$ 


$$
\begin{aligned}
M_{\gamma}(s)= & \left(\Gamma\left(m_{1}\right) \Gamma\left(\left\lceil m_{1}\right\rceil-m_{1}\right) \Gamma\left(m_{2}\right)\right)^{-1} \sum_{k=0}^{\left\lceil m_{1}\right\rceil}\left(\begin{array}{c}
\left\lceil m_{1}\right\rceil \\
k
\end{array}\right)\left(\frac{\bar{\gamma}_{2}}{c m_{2}}\right)^{k} \\
& \times G_{1,[1: 1], 0,[1: 1]}^{1,1,1,1,1}\left[\begin{array}{c|c}
\frac{\left(m_{1}+s \bar{\gamma}_{1}\right) \bar{\gamma}_{2}}{c m_{1} m_{2}} & 1-m_{1} ; 1-\left\lceil m_{1}\right\rceil+m_{1} \\
\frac{\bar{\gamma}_{2}}{c m_{2}} & - \\
\end{array}\right] .
\end{aligned}
$$

fading channels is given by (9) at the top of this page, where $\lceil$.$\rceil stands for the integer ceiling operator and G[. \mid$.$] denotes$ the generalized Meijer's $G$-function of two variables defined in (31) of Appendix A.

Proof: See Appendix B.

Although the $G$-function in (9) cannot be directly calculated by popular mathematical softwares such as Matlab and Mathematica, it can be easily evaluated by the algorithm recently developed in [25, Table II], which is based on the double Mellin-Barnes type integrals. On the other hand, in order to show more extensive applications of Theorem 1, we will derive an analytical expansion for the MGF in the next subsection.

Special Case: When $m_{1}$ takes integer values, that is, $\left\lceil m_{1}\right\rceil-$ $m_{1}=0$, the generalized $G$-function of two variables in (9) reduces to the conventional Meijer's $G$-function. Specifically, we have the following proposition.

Proposition 1: For $b_{1}^{\prime}=1$ and $x \neq 0$, the generalized $G$ function of two variables reduces to the conventional Meijer's $G$-function of one variable, that is,

$$
G_{1,11: 1], 0,[1: 1]}^{1,1,1,1}\left[\begin{array}{c|c}
x & a_{1} \\
b_{1} ; b_{1}^{\prime} \\
- \\
0 ; 0
\end{array}\right]=G_{1,2}^{2,1}\left(\frac{1}{x} \mid \begin{array}{c}
1 \\
a_{1}, 1-b_{1}
\end{array}\right) .
$$

Proof: See Appendix C.

Based on Proposition 1, it is straightforward to show that when $m_{1}$ takes integer values, (9) reduces to

$$
\begin{aligned}
M_{\gamma}(s)= & \frac{1}{\Gamma\left(m_{1}\right) \Gamma\left(m_{2}\right)} \sum_{k=0}^{m_{1}}\left(\begin{array}{c}
m_{1} \\
k
\end{array}\right)\left(\frac{\bar{\gamma}_{2}}{c m_{2}}\right)^{k} \\
& \times G_{1,2}^{2,1}\left(\frac{c m_{1} m_{2}}{\left(m_{1}+s \bar{\gamma}_{1}\right) \bar{\gamma}_{2}} \mid \begin{array}{c}
1 \\
k+m_{2}, m_{1}
\end{array}\right)
\end{aligned}
$$

which is equivalent to the result reported in [19, Eq. (8)].

\section{B. PDF of the End-to-End SNR}

Although the MGF is explicitly given in (9), the PDF cannot be directly obtained by applying the inverse Laplace transform due to the high complexity of the involved $G$-function. In order to proceed, we derive an analytical expansion for the MGF. More specifically, from the expression in (31) of Appendix A and by using the residue theorem, this $G$-function can be equivalently evaluated by considering the residues at the poles of the integrand in (31) [23, p. 538]. Therefore, the MGF in
(9) can be reformulated using [23, Eq. (2.3)], thus yielding

$$
\begin{aligned}
M_{\gamma}(s)= & \sum_{k=0}^{\left\lceil m_{1}\right\rceil}\left(\begin{array}{c}
\left\lceil m_{1}\right\rceil \\
k
\end{array}\right)\left(\frac{\bar{\gamma}_{2}}{c m_{2}}\right)^{k}\left(m_{2}\right)_{k} \\
& \times \sum_{p=0}^{\infty} \sum_{q=0}^{\infty} \frac{\left(k+m_{2}\right)_{p+q}}{p ! q !}\left(m_{1}\right)_{p}\left(\left\lceil m_{1}\right\rceil-m_{1}\right)_{q} \\
& \times\left(-\frac{\left(m_{1}+s \bar{\gamma}_{1}\right) \bar{\gamma}_{2}}{c m_{1} m_{2}}\right)^{p}\left(-\frac{\bar{\gamma}_{2}}{c m_{2}}\right)^{q}
\end{aligned}
$$

where $(a)_{k}=\Gamma(a+k) / \Gamma(a)$ is the Pochhammer symbol. Then, exploiting the symbolic operators proposed in [27], it can be shown that the double series in (12) can be transformed into a single series of the product of two generalized hypergeometric functions and, thus, the MGF can be reformulated as (13)-(14) at the top of next page, where we exploited the identity ${ }_{2} F_{0}(a, b ;-;-1 / x)=x^{a} \Psi(a ; 1+a-b ; x)$ [28, Eq. (13.1.10)] in (14). Finally, performing the inverse Laplace transform of (14) leads to the PDF of the end-to-end SNR as summarized in the following corollary, where we exploited [21, vol. 5, Eq. (3.34.1.9)] as well as the scaling and shifting properties of the inverse Laplace transform.

Corollary 1: The PDF of the end-to-end SNR of dualhop semi-blind AF relaying systems over arbitrary Nakagami$m$ fading channels is given by (15) in the middle of next page, where ${ }_{p} F_{q}($.$) stands for the generalized hypergeometric$ function [26, Eq. (4.1.(1))].

\section{Moments of the End-to-End SNR}

Although the moments of the received SNR can be obtained by taking the derivatives of the MGF in (9) and setting $s=0$, the resultant expressions are extremely complicated. On the other hand, the moments with a simple form can be calculated in a straightforward manner and they are summarized in the following lemma.

Lemma 1: The $n^{\text {th }}$-order moment of the end-to-end SNR of dual-hop semi-blind AF relaying systems is given by

$$
\begin{aligned}
\mathbb{E}\left\{\gamma^{n}\right\}= & \left(m_{1}\right)_{n}\left(m_{2}\right)_{n}\left(\frac{\bar{\gamma}_{1}}{m_{1}}\right)^{n}\left(\frac{c m_{2}}{\bar{\gamma}_{2}}\right)^{m_{2}} \\
& \times \Psi\left(m_{2}+n, m_{2}+1 ; \frac{c m_{2}}{\bar{\gamma}_{2}}\right) .
\end{aligned}
$$

Proof: See Appendix D.

For the ease of subsequent discussion, the first-order and second-order moments of the end-to-end SNR are explicitly expressed as follows.

Corollary 2: The first-order and second-order moments of the end-to-end SNR of dual-hop semi-blind AF relaying 


$$
\begin{aligned}
& M_{\gamma}(s)=\sum_{k=0}^{\left\lceil m_{1}\right\rceil}\left(\begin{array}{c}
\left\lceil m_{1}\right\rceil \\
k
\end{array}\right)\left(\frac{\bar{\gamma}_{2}}{c m_{2}}\right)^{k}\left(m_{2}\right)_{k} \sum_{r=0}^{\infty} \frac{1}{r !}\left(k+m_{2}\right)_{r}\left(m_{1}\right)_{r}\left(\left\lceil m_{1}\right\rceil-m_{1}\right)_{r} \\
& \times\left(\frac{\bar{\gamma}_{2}}{c m_{2}}\right)^{r}{ }_{2} F_{0}\left(k+m_{2}+r,\left\lceil m_{1}\right\rceil-m_{1}+r ;-;-\frac{\bar{\gamma}_{2}}{c m_{2}}\right) \\
& \times\left(\frac{\left(m_{1}+s \bar{\gamma}_{1}\right) \bar{\gamma}_{2}}{c m_{1} m_{2}}\right)^{r}{ }_{2} F_{0}\left(k+m_{2}+r, m_{1}+r ;-;-\frac{\left(m_{1}+s \bar{\gamma}_{1}\right) \bar{\gamma}_{2}}{c m_{1} m_{2}}\right) \\
& =\sum_{k=0}^{\left\lceil m_{1}\right\rceil}\left(\begin{array}{c}
\left\lceil m_{1}\right\rceil \\
k
\end{array}\right) m_{1}^{k+m_{2}}\left(m_{2}\right)_{k} \sum_{r=0}^{\infty} \frac{1}{r !}\left(k+m_{2}\right)_{r}\left(m_{1}\right)_{r}\left(\left\lceil m_{1}\right\rceil-m_{1}\right)_{r} \\
& \times\left(\frac{c m_{2}}{\bar{\gamma}_{2}}\right)_{2}^{m_{2}-r} F_{0}\left(k+m_{2}+r,\left\lceil m_{1}\right\rceil-m_{1}+r ;-;-\frac{\bar{\gamma}_{2}}{c m_{2}}\right) \\
& \times \frac{1}{\left(m_{1}+s \bar{\gamma}_{1}\right)^{k+m_{2}}} \Psi\left(k+m_{2}+r, 1+k-m_{1}+m_{2} ; \frac{c m_{1} m_{2}}{\left(m_{1}+s \bar{\gamma}_{1}\right) \bar{\gamma}_{2}}\right) \text {. }
\end{aligned}
$$

$$
\begin{aligned}
f_{\gamma}(\gamma)= & \sum_{k=0}^{\left\lceil m_{1}\right\rceil}\left(\begin{array}{c}
\left\lceil m_{1}\right\rceil \\
k
\end{array}\right) m_{1}^{k+m_{2}}\left(m_{2}\right)_{k} \sum_{r=0}^{\infty} \frac{1}{r !}\left(k+m_{2}\right)_{r}\left(m_{1}\right)_{r}\left(\left\lceil m_{1}\right\rceil-m_{1}\right)_{r} \\
& \times\left(\frac{c m_{2}}{\bar{\gamma}_{2}}\right)^{m_{2}-r}{ }_{2} F_{0}\left(k+m_{2}+r,\left\lceil m_{1}\right\rceil-m_{1}+r ;-;-\frac{\bar{\gamma}_{2}}{c m_{2}}\right) \\
& \times \frac{1}{\bar{\gamma}_{1}} \exp \left(-\frac{m_{1}}{\bar{\gamma}_{1}} \gamma\right)\left[\frac{\Gamma\left(-k+m_{1}-m_{2}\right)}{\Gamma\left(r+m_{1}\right) \Gamma\left(k+m_{2}\right)}\left(\frac{\gamma}{\bar{\gamma}_{1}}\right)^{k+m_{2}-1}\right. \\
& \times{ }_{1} F_{2}\left(k+m_{2}+r ; 1+k-m_{1}+m_{2}, k+m_{2} ; \frac{c m_{1} m_{2}}{\bar{\gamma}_{1} \bar{\gamma}_{2}} \gamma\right) \\
& +\frac{\Gamma\left(k-m_{1}+m_{2}\right)}{\Gamma\left(k+m_{2}+r\right) \Gamma\left(m_{1}\right)}\left(\frac{c m_{1} m_{2}}{\bar{\gamma}_{2}}\right)^{-k+m_{1}-m_{2}}\left(\frac{\gamma}{\bar{\gamma}_{1}}\right)^{m_{1}-1} \\
& \left.\times{ }_{1} F_{2}\left(r+m_{1} ; 1-k+m_{1}-m_{2}, m_{1} ; \frac{c m_{1} m_{2}}{\bar{\gamma}_{1} \bar{\gamma}_{2}} \gamma\right)\right] .
\end{aligned}
$$

systems are given by

$$
\begin{aligned}
\mathbb{E}\{\gamma\}= & m_{2} \bar{\gamma}_{1}\left(\frac{c m_{2}}{\bar{\gamma}_{2}}\right)^{m_{2}} \\
& \times \Psi\left(m_{2}+1, m_{2}+1 ; \frac{c m_{2}}{\bar{\gamma}_{2}}\right)
\end{aligned}
$$

and

$$
\begin{aligned}
\mathbb{E}\left\{\gamma^{2}\right\}= & \frac{m_{2}}{m_{1}}\left(m_{1}+1\right)\left(m_{2}+1\right) \bar{\gamma}_{1}^{2}\left(\frac{c m_{2}}{\bar{\gamma}_{2}}\right)^{m_{2}} \\
& \times \Psi\left(m_{2}+2, m_{2}+1 ; \frac{c m_{2}}{\bar{\gamma}_{2}}\right)
\end{aligned}
$$

respectively.

Proof: The results can be proved by substituting $n=1$ and $n=2$ into (16), and with straightforward manipulations.

\section{Further Performance Analyses}

After obtaining the MGF, PDF and moments of the end-toend SNR, we investigate in this section the outage probability, average SEP, ergodic capacity and diversity order of the dualhop semi-blind AF relay transmission.

\section{A. Outage Probability}

The outage probability is defined as the probability that the instantaneous SNR falls below a predefined threshold value $\gamma_{\mathrm{th}}$. Mathematically, the outage probability is expressed as

$$
\mathrm{P}_{\text {outage }}\left(\gamma_{\mathrm{th}}\right)=\operatorname{Pr}\left\{\gamma<\gamma_{\mathrm{th}}\right\}=\int_{0}^{\gamma_{\text {th }}} f_{\gamma}(\gamma) \mathrm{d} \gamma
$$

In order to obtain a closed-form expression of (19), we first rewrite the PDF in (15) by applying the infinite series expansion of the exponential function [21, vol. 1, Eq. (5.2.7.2)]. Then, substituting the formulation of (15) into (19) and making use of [21, vol. 3, Eq. (2.22.2.1)], after some manipulations, we finally obtain (20) at the top of next page, where $B(a, b)$ is the Beta function. Although it seems complicated, (20) mainly involves common Gamma function, Beta function and generalized hypergeometric function. Hence, (20) is easy to be numerically evaluated and its accuracy is corroborated by the simulation results in Section $\mathrm{V}$.

In [19], an approximate analysis for the performance of dual-hop AF relaying over Nakagami- $m$ fading channels with non-integer $m$ values is presented, based on an upper bound 


$$
\begin{aligned}
\mathrm{P}_{\text {outage }}\left(\gamma_{\mathrm{th}}\right)= & \sum_{k=0}^{\left\lceil m_{1}\right\rceil}\left(\begin{array}{c}
\left\lceil m_{1}\right\rceil \\
k
\end{array}\right) m_{1}^{k+m_{2}}\left(m_{2}\right)_{k} \sum_{r=0}^{\infty} \frac{1}{r !}\left(k+m_{2}\right)_{r}\left(m_{1}\right)_{r}\left(\left\lceil m_{1}\right\rceil-m_{1}\right)_{r} \\
& \times\left(\frac{c m_{2}}{\bar{\gamma}_{2}}\right)^{m_{2}-r}{ }_{2} F_{0}\left(k+m_{2}+r,\left\lceil m_{1}\right\rceil-m_{1}+r ;-;-\frac{\bar{\gamma}_{2}}{c m_{2}}\right) \\
& \times \sum_{p=0}^{\infty} \frac{\left(-m_{1}\right)^{p}}{p !}\left[\frac{\Gamma\left(-k+m_{1}-m_{2}\right)}{\Gamma\left(r+m_{1}\right) \Gamma\left(k+m_{2}\right)}\left(\frac{\gamma_{t h}}{\bar{\gamma}_{1}}\right)^{p+k+m_{2}} B\left(p+k+m_{2}, 1\right)\right. \\
& \times{ }_{2} F_{3}\left(k+m_{2}+r, p+k+m_{2} ; 1+k-m_{1}+m_{2}, k+m_{2}, p+k+m_{2}+1 ; \frac{c m_{1} m_{2}}{\bar{\gamma}_{1} \bar{\gamma}_{2}} \gamma_{t h}\right) \\
& +\frac{\Gamma\left(k-m_{1}+m_{2}\right)}{\Gamma\left(k+m_{2}+r\right) \Gamma\left(m_{1}\right)}\left(\frac{c m_{1} m_{2}}{\bar{\gamma}_{2}}\right)^{-k+m_{1}-m_{2}}\left(\frac{\gamma_{t h}}{\bar{\gamma}_{1}}\right)^{p+m_{1}} B\left(p+m_{1}, 1\right) \\
& \left.\times{ }_{2} F_{3}\left(r+m_{1}, p+m_{1} ; 1-k+m_{1}-m_{2}, m_{1}, p+m_{1}+1 ; \frac{c m_{1} m_{2}}{\bar{\gamma}_{1} \bar{\gamma}_{2}} \gamma_{t h}\right)\right]
\end{aligned}
$$

on the end-to-end SNR [19, Eq. (25)]:

$$
\gamma \leq \min \left\{\gamma_{1}, \frac{1}{c} \gamma_{1} \gamma_{2}\right\}
$$

For the purpose of comparison, the lower bound on the outage probability is reproduced as follows [19, Eq. (28)]:

$$
\begin{aligned}
\mathrm{P}_{\text {outage }}^{\mathrm{L}}\left(\gamma_{\mathrm{th}}\right)= & \frac{1}{\Gamma\left(m_{1}\right)} \gamma\left(m_{1}, \frac{m_{1}}{\bar{\gamma}_{1}} \gamma_{\mathrm{th}}\right)+\frac{1}{\Gamma\left(m_{1}\right) \Gamma\left(m_{2}\right)} \\
& \times \sum_{k=0}^{\infty} \frac{(-1)^{k}}{\left(m_{2}+k\right) k !}\left(\frac{c m_{1} m_{2}}{\bar{\gamma}_{1} \bar{\gamma}_{2}} \gamma_{\mathrm{th}}\right)^{m_{2}+k} \\
& \times \Gamma\left(m_{1}-m_{2}-k, \frac{m_{1}}{\bar{\gamma}_{1}} \gamma_{\mathrm{th}}\right)
\end{aligned}
$$

where $\gamma(a, x)=\int_{0}^{x} t^{a-1} e^{-t} \mathrm{~d} t$ denotes the lower incomplete Gamma function and $\Gamma(a, x)=\int_{x}^{\infty} t^{a-1} e^{-t} \mathrm{~d} t$ stands for the upper incomplete Gamma function. The accuracy of (22) compared to (20) will be illustrated in Figs. 1-3, and is detailed in Section V.

\section{B. Average Symbol Error Probability}

By exploiting the MGF in (9), it is straightforward to obtain the average SEP of different kinds of modulation schemes [29], [30]. Herein, we focus on the $M$-PSK constellation since it is adopted in 3GPP long-term evolution (LTE) systems [31]. For the coherently detected $M$-PSK, the average SEP is given by [20, Eq. (8.23)]

$$
\mathrm{P}_{\mathrm{S}}(\mathrm{E})=\frac{1}{\pi} \int_{0}^{\Theta} M_{\gamma}\left(\frac{g_{\mathrm{PSK}}}{\sin ^{2} \theta}\right) \mathrm{d} \theta
$$

where the constants $g_{\mathrm{PSK}}=\sin ^{2}(\pi / M)$ and $\Theta=(M-$ 1) $\pi / M$. Although its closed-form expression cannot be obtained, (23) can be easily evaluated numerically since it is a proper integral with finite limits and closed-form integrand.

On the other hand, a closed-form approximation of the average SEP can be obtained by substituting the MGF into the following expression [32, Eq. (10)]

$$
\mathrm{P}_{\mathrm{S}}(\mathrm{E}) \approx\left(\frac{\Theta}{2 \pi}-\frac{1}{6}\right) M_{\gamma}\left(g_{\mathrm{PSK}}\right)+\frac{1}{4} M_{\gamma}\left(\frac{4}{3} g_{\mathrm{PSK}}\right)
$$

$$
+\left(\frac{\Theta}{2 \pi}-\frac{1}{4}\right) M_{\gamma}\left(\frac{g_{\mathrm{PSK}}}{\sin ^{2} \Theta}\right) .
$$

For the numerical evaluation of (23) and (24), (14) is exploited, which mainly involves the generalized hypergeometric function and can be easily calculated. The accuracy of (23) and (24) will be demonstrated in Section V.

\section{Ergodic Capacity}

The ergodic capacity is defined as the statistical mean of the instantaneous mutual information between the source and the destination, in the unit of bit/s/Hz. Mathematically,

$$
C_{\text {erg }}=\frac{1}{2} \int_{0}^{\infty} \log _{2}(1+\gamma) f_{\gamma}(\gamma) \mathrm{d} \gamma
$$

which can be numerically evaluated by exploiting the analytical expression of $f_{\gamma}(\gamma)$ in (15). The factor $1 / 2$ is introduced by the fact that two transmission phases are involved.

On the other hand, in view of the moments of the end-to-end SNR in (16), a closed-form expression of the ergodic capacity can be obtained by exploiting the Taylor series expansion of $\log _{2}(1+\gamma)$ with respect to $1+\mathbb{E}\{\gamma\}$. More specifically,

$$
\begin{aligned}
& C_{\mathrm{erg}}=\frac{1}{2 \ln (2)} \mathbb{E}\{\ln (1+\gamma)\} \\
& =\frac{1}{2 \ln (2)} \ln (1+\mathbb{E}\{\gamma\}) \\
& +\frac{1}{2 \ln (2)} \mathbb{E}\left\{\sum_{n=1}^{\infty} \frac{(\gamma-\mathbb{E}\{\gamma\})^{n}}{n !} \ln ^{(n)}(1+\mathbb{E}\{\gamma\})\right\} \\
& =\frac{1}{2 \ln (2)} \ln (1+\mathbb{E}\{\gamma\}) \\
& -\frac{\mathbb{E}\left\{\gamma^{2}\right\}-\mathbb{E}^{2}\{\gamma\}}{4 \ln (2)(1+\mathbb{E}\{\gamma\})^{2}}+o\left[(\gamma-\mathbb{E}\{\gamma\})^{3}\right],
\end{aligned}
$$

where $o[$.$] denotes the Landau notation and \mathbb{E}\{\gamma\}$ and $\mathbb{E}\left\{\gamma^{2}\right\}$ are explicitly shown in (17) and (18), respectively. The accuracy of the numerical integration (25) and its approximation (26) will be examined in Section $\mathrm{V}$, in comparison with the simulation results.

Here it is worth mentioning that a unified MGF-based approach for computing the ergodic capacity over generalized 
fading channels was recently developed in [33, Eq. (7)]. Unfortunately, the unified approach cannot be applied here, due to the complicated structure of the MGF in (14), and it is expected that no closed-form capacity expression can be derived in terms of conventional special functions. On the other hand, notice that, although the Taylor formula is also exploited in [9, Eq. (6)], the ergodic capacity analysis there cannot be applied in the general Nakagami- $m$ fading case with non-integer $m$ values, given that the finite-sum expansion of the incomplete Gamma function was exploited in [9, Eq. (18)]. Moreover, since the PDF of the end-to-end SNR is not provided in [9], the ergodic capacity cannot be directly evaluated using (25).

\section{Diversity Order}

In order to gain more insight into the MGF, the diversity order is derived in this section. Diversity order is an important measure of the bit/symbol error rate performance at high SNR. It can be found by using the asymptotic expression of the MGF $M_{\gamma}(s)$ as $s \rightarrow \infty$. More specifically, as $s \rightarrow \infty, M_{\gamma}(s)$ can be written in the form

$$
\left|M_{\gamma}(s)\right|=a|s|^{-b}+o\left(|s|^{-b}\right),
$$

then the diversity order is $b$ [34]. The MGF formulation (14) can be exploited to derive the diversity order. In particular, we focus on the asymptotic expression of the Tricomi hypergeometric function in (14) for the following two cases.

1) $1+k-m_{1}+m_{2}>1$ : In this case, as $s \rightarrow \infty$, the Tricomi hypergeometric function in (14) is asymptotically given by [35, Eq. (10.30a)]:

$$
\begin{aligned}
& \Psi\left(k+m_{2}+r, 1+k-m_{1}+m_{2} ; \frac{c m_{1} m_{2}}{\left(m_{1}+s \bar{\gamma}_{1}\right) \bar{\gamma}_{2}}\right) \\
& \sim \frac{\Gamma\left(k-m_{1}+m_{2}\right)}{\Gamma\left(k+m_{2}+r\right)}\left(\frac{c m_{1} m_{2}}{\left(m_{1}+s \bar{\gamma}_{1}\right) \bar{\gamma}_{2}}\right)^{m_{1}-m_{2}-k} .
\end{aligned}
$$

Substituting (28) into (14), it is straightforward to show that the exponent of the term $m_{1}+s \bar{\gamma}_{1}$ is $-m_{1}$. Therefore, the diversity order is $m_{1}$ according to (27).

2) $1+k-m_{1}+m_{2}<1$ : In this case, as $s \rightarrow \infty$, we get

$$
\begin{aligned}
& \Psi\left(k+m_{2}+r, 1+k-m_{1}+m_{2} ; \frac{c m_{1} m_{2}}{\left(m_{1}+s \bar{\gamma}_{1}\right) \bar{\gamma}_{2}}\right) \\
& =\left(\frac{c m_{1} m_{2}}{\left(m_{1}+s \bar{\gamma}_{1}\right) \bar{\gamma}_{2}}\right)^{m_{1}-m_{2}-k} \\
& \times \Psi\left(m_{1}+r, 1-k+m_{1}-m_{2} ; \frac{c m_{1} m_{2}}{\left(m_{1}+s \bar{\gamma}_{1}\right) \bar{\gamma}_{2}}\right) \\
& \sim \frac{\Gamma\left(m_{1}-m_{2}-k\right)}{\Gamma\left(m_{1}+r\right)},
\end{aligned}
$$

where we exploited [35, Eq. (10.25)] to reach (29) and [35, Eq. (10.30a)] was used again to obtain (30). Substituting (30) into (14) and in view of (27), we obtain a diversity order of $m_{2}$. Combining the two cases above, we conclude that the diversity order is $\min \left\{m_{1}, m_{2}\right\}$, which implies that the weakest hop dominates the bit/symbol error rate performance. This conclusion reduces to the well-known result in [3] where integer $m_{1}$ and $m_{2}$ are assumed. The effect of diversity order

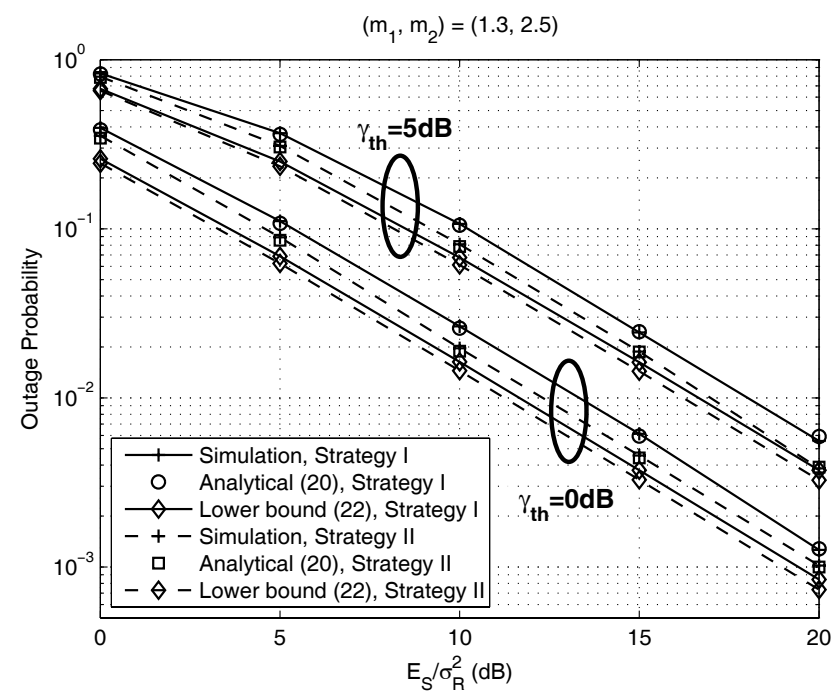

Fig. 1. Outage probability of the dual-hop semi-blind AF relaying transmission with fading parameters $\left(m_{1}, m_{2}\right)=(1.3,2.5)$.

on the slope of the SEP curves will be illustrated in the next section.

\section{Numerical EXAMPLES AND Discussions}

In this section, numerical examples for the above analyses are presented and corroborated by simulation results. In all simulations, without loss of generality, the variances of the AWGN at the relay and at the destination are assumed to be identical, that is, $\sigma_{R}^{2}=\sigma_{D}^{2}$. Any infinite series involved in the analytical computation, such as the MGF in (14) and the outage probability in (20), are calculated through its first 6 terms.

Figure 1 depicts the outage probability versus $E_{S} / \sigma_{R}^{2}$ in $\mathrm{dB}$ with $\left(m_{1}, m_{2}\right)=(1.3,2.5)$. That is, the channel fading of the fist hop is a little worse than the second hop. The outage threshold $\gamma_{\text {th }}$ is set to 0 and $5 \mathrm{~dB}$. It is observed that the numerical results of (20) match perfectly with the simulation results. Moreover, as expected, the outage probability decreases with increasing $E_{S} / \sigma_{R}^{2}$, but increases with higher threshold values. On the other hand, the lower bound on the outage probability in (22) is loose in the whole SNR range under consideration.

Figure 2 is generated based on the same simulation settings as Fig. 1 except that the fading parameters are changed to $\left(m_{1}, m_{2}\right)=(2.6,1.2)$. That is, the second hop experiences more severe fading than the first hop. It is seen that the numerical results of (20) still agree perfectly with simulation results. On the other hand, the lower bound on the outage probability in (22) is loose at low SNR and it becomes tight at high SNR.

Furthermore, two different strategies to compute the relay gain in Section III-A are compared in Figs. 1 and 2. It is observed that Strategy II slightly outperforms Strategy I. This is because the calculation of the relay gain in (6) and (7) differs only in the position of the expectation operator and the fact that the function $1 /\left(\gamma_{1}+1\right)$ is a strictly convex function, such that $\beta_{I I}^{2}$ in (7) is always greater than $\beta_{I}^{2}$ in (6). 


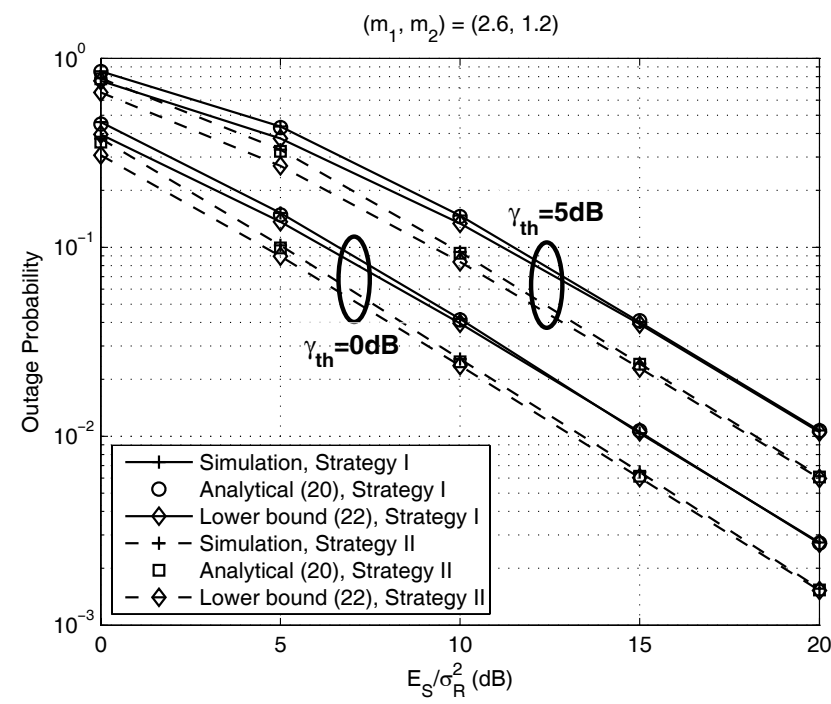

Fig. 2. Outage probability of the dual-hop semi-blind AF relaying transmission with fading parameters $\left(m_{1}, m_{2}\right)=(2.6,1.2)$.

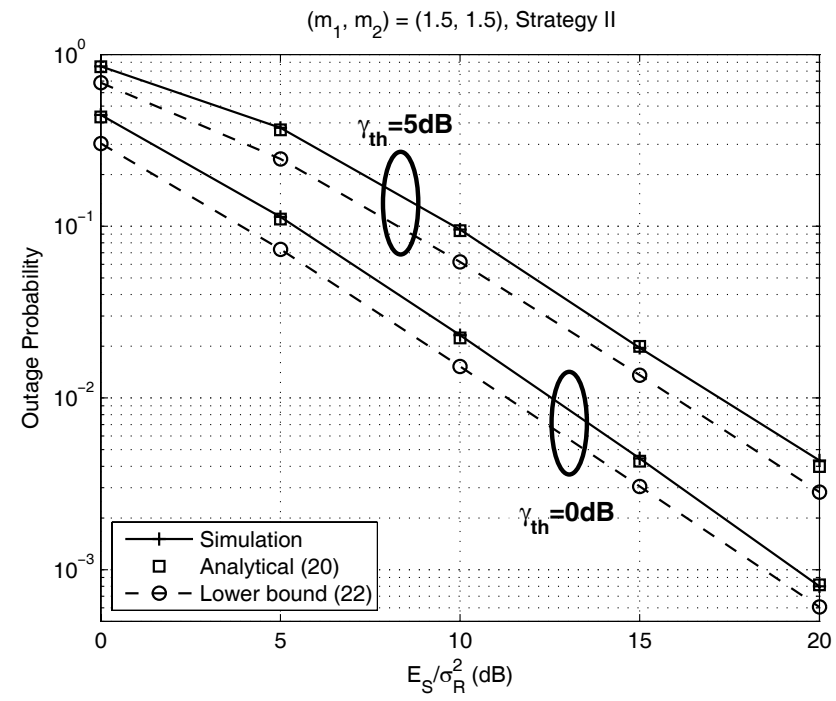

Fig. 3. Outage probability of the dual-hop semi-blind AF relaying transmission with fading parameters $\left(m_{1}, m_{2}\right)=(1.5,1.5)$.

The accuracy advantage of the closed-form outage probability in (20) over that of the lower bound in (22) is further illustrated in Fig. 3, for the symmetric fading case with $\left(m_{1}, m_{2}\right)=(1.5,1.5)$. It is observed that (22) is loose in the whole SNR range under consideration. Combining the observations in Figs. 1-3, we conclude that it is only when $m_{1} \gg m_{2}$ and the SNR is high that the lower bound on the outage probability in (22) becomes tight. This is because the upper bound on the end-to-end SNR in (21) performs well only when $\gamma_{1} \gg \gamma_{2}$ and the SNR is high.

Figure 4 illustrates the average SEP of semi-blind AF relaying transmission with Strategy $I$ versus $E_{S} / \sigma_{R}^{2}$ in $\mathrm{dB}$. QPSK constellation is applied in the simulations, that is, $M=4$. It is observed that the numerical results of (23) and (24) agree perfectly with the simulation results. Figure 5 shows the average SEP with Strategy II, where the simulation settings are the same as those in Fig. 4. It is seen that (23) and (24) again agree exactly with the simulation results.

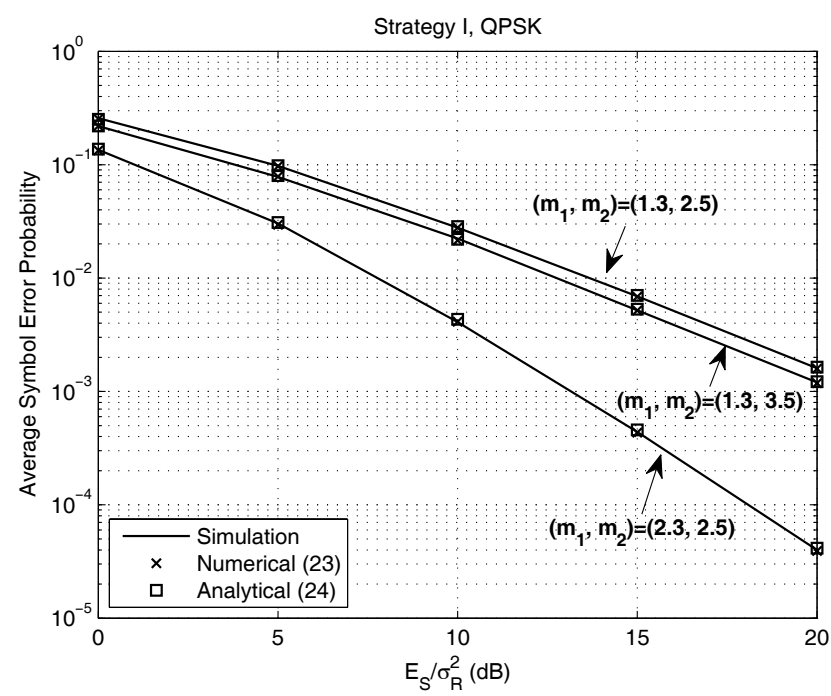

Fig. 4. Average SEP of the dual-hop semi-blind AF relaying transmission with Strategy I.

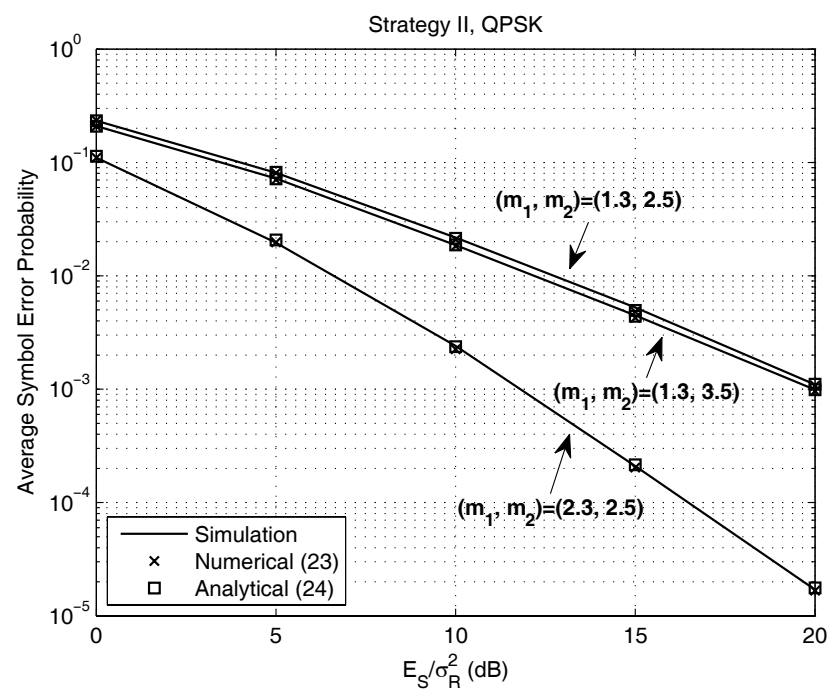

Fig. 5. Average SEP of the dual-hop semi-blind AF relaying transmission with Strategy II.

It is observed from Fig. 4 that the curve with $\left(m_{1}, m_{2}\right)=$ $(1.3,2.5)$ has the same slope as the curve with $\left(m_{1}, m_{2}\right)=$ $(1.3,3.5)$. On the other hand, the curve with $\left(m_{1}, m_{2}\right)=$ $(2.3,2.5)$ has steeper slope than that of both curves above. These observations agree with the result in Section IV-D, namely, the diversity order is $\min \left\{m_{1}, m_{2}\right\}$. Also, comparing Fig. 4 and Fig. 5, we observe that Strategies I and II have the same diversity order for a given $m_{1}$ and $m_{2}$. This is consistent with the fact that our analyses apply to all AF relaying schemes with fixed gain.

Figure 6 shows the ergodic capacity with Strategy I versus $E_{S} / \sigma_{R}^{2}$ in dB. It is observed that the integral expression (25) agrees exactly with the simulation results. Also, the closedform approximation (26) match the simulation results very well. As expected, the ergodic capacity increases with larger $m_{1}$ and $m_{2}$. Finally, the ergodic capacity of Strategy II is illustrated in Fig. 7, where the simulation settings are the same as those in Fig. 6. It is observed that the numerical results of 


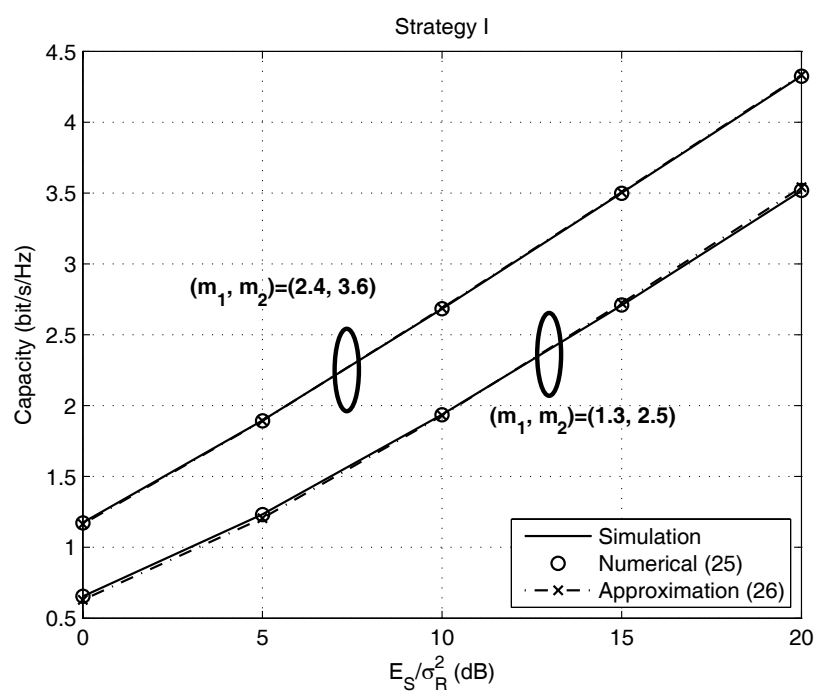

Fig. 6. Ergodic capacity of the dual-hop semi-blind AF relaying transmission with Strategy I.

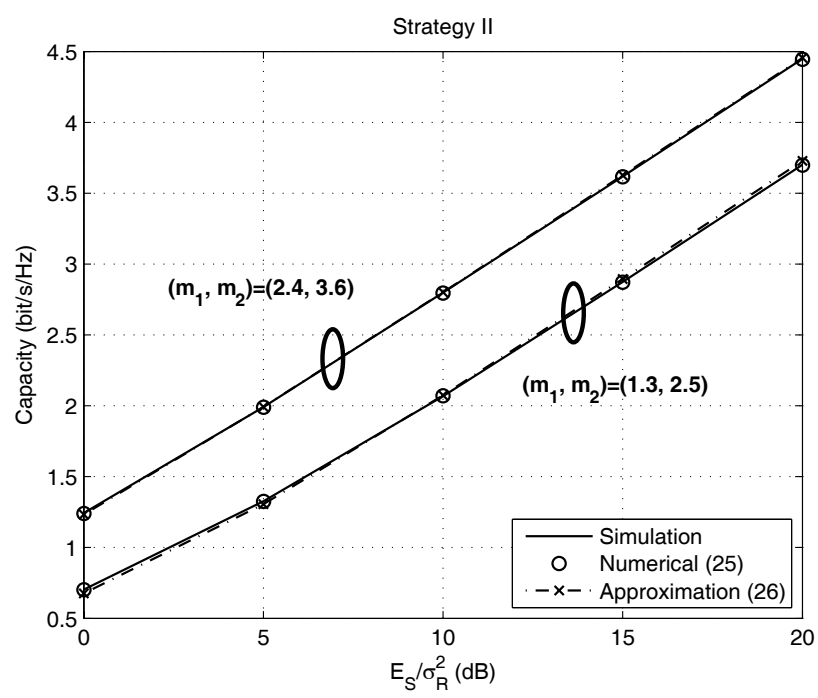

Fig. 7. Ergodic capacity of the dual-hop semi-blind AF relaying transmission with Strategy II.

(25) and the approximation results of (26) still agree perfectly with the simulation results.

\section{CONCLUSION}

The MGF, PDF and moments of the end-to-end SNR of dual-hop semi-blind AF relay transmission over arbitrary Nakagami- $m$ fading channels were explicitly derived in this paper. With these results, the system performance in terms of outage probability, average SEP, ergodic capacity and diversity order, were analytically obtained. The analysis developed in this work applies to any semi-blind AF relaying transmission with fixed relay gain. All analytical results were demonstrated to be efficient tools to accurately evaluate system performance.

\section{APPENDIX A}

Definition of the Generalized MeIJeR's $G$-FunCtion OF TwO VARIABLES

The generalized Meijer's $G$-function of two variables is defined in terms of double Mellin-Barnes type integrals as (31) at the top of next page [22, Eq. (1.1)].

The paths of integration $L_{1}$ and $L_{2}$ can be identified such that the poles of $\Gamma\left(\beta_{j}+\xi\right), j=1, \cdots, m_{1}$ and $\Gamma\left(\beta_{j}^{\prime}+\eta\right)$, $j=1, \cdots, m_{2}$ lie to the right, and those of $\Gamma\left(\gamma_{j}-\xi\right), j=$ $1, \cdots, v_{1}, \Gamma\left(\gamma_{j}^{\prime}-\eta\right), j=1, \cdots, v_{2}$ and $\Gamma\left(1-\varepsilon_{j}-\xi-\eta\right)$, $j=1, \cdots, n$ lie to the left of imaginary axis [23, p. 538]. Notice that, the integral in (31) and therefore the Meijer's $G$ function of two variables converges if the following conditions are satisfied [22, p. 62]:

$$
\begin{gathered}
p+q_{1}+s+t_{1}<2\left(m_{1}+v_{1}+n\right), \\
p+q_{2}+s+t_{2}<2\left(m_{2}+v_{2}+n\right), \\
|\arg (x)|<\pi\left[m_{1}+v_{1}+n-\left(p+q_{1}+s+t_{1}\right) / 2\right], \\
|\arg (y)|<\pi\left[m_{2}+v_{2}+n-\left(p+q_{2}+s+t_{2}\right) / 2\right] .
\end{gathered}
$$

It is straightforward to show that the parameters of the $G$ function in (9) satisfy these sufficient conditions above, and therefore the $G$-function converges in the sense that it is of finite value.

\section{APPENDIX B \\ PROOF OF THEOREM 1}

The MGF of the end-to-end SNR in (3) can be calculated as (36)-(38) in the middle of next page, where we exploited [21, vol. 1, Eq. (2.3.3.1)] in (37) and the binomial expansion theorem in (38). Furthermore, the integration term in (38) can be calculated as (39)-(42) at the bottom of next page, where the constant $C \triangleq 1 /\left(\Gamma\left(m_{1}\right) \Gamma\left(\left\lceil m_{1}\right\rceil-m_{1}\right)\right)$ and we exploited the equality $(1+z)^{-\alpha}=\frac{1}{\Gamma(\alpha)} H_{1,1}^{1,1}\left[z \mid \begin{array}{l}(1-\alpha, 1) \\ (0,1)\end{array}\right], \alpha \geq 0[24$, p. 152] in (40) with $H_{1,1}^{1,1}[. \mid$.] being the Fox's $H$-function [24, Eq. (1.1.1)]. The Laplace transform of the product of two $H$ functions [24, Eq. (2.6.2)] was applied to reach (41), which holds for any $m_{1}, m_{2}, c, \bar{\gamma}_{1}$ and $\bar{\gamma}_{2}>0$. These parameter constraints are obviously true for the Nakagami- $m$ fading channels under consideration. The function $H_{1,[1: 1], 0,[1: 1]}^{1,1, \mid .]}$ denotes the generalized $H$-function of two variables and it reduces to the generalized $G$-function of two variables in (42) with the help of [24, Eq. (2.3.1)]. Finally, substituting (42) into (38) and performing some mathematical manipulations yields (9).

\section{APPENDIX C \\ PROOF OF PROPOSITION 1}

Using the series expression of the generalized $G$-function of two variables and putting $b_{1}^{\prime}=1$, we have [23, Eq. (2.3)]

$$
G_{1,[1: 1], 0,[1: 1]}^{1,1,1,1}\left[\begin{array}{c|c}
x & a_{1} \\
b_{1} ; 1 \\
- \\
0 ; 0
\end{array}\right]
$$




$$
\begin{aligned}
& G_{\left.p, t_{1}: t_{2}\right], s,\left[q_{1}: q_{2}\right]}^{n, v_{1}, m_{1}, m_{2}}\left[\begin{array}{c|c}
\left(\varepsilon_{p}\right) \\
y \\
\left(\gamma_{t_{1}}\right) ;\left(\gamma_{t_{2}}^{\prime}\right) \\
\left(\delta_{s}\right) \\
\left(\beta_{v_{1}}\right) ;\left(\beta_{v_{2}}^{\prime}\right)
\end{array}\right] \\
& =\frac{-1}{4 \pi^{2}} \int_{L_{2}} \int_{L_{1}} \frac{\prod_{j=1}^{m_{1}} \Gamma\left(\beta_{j}+\xi\right) \prod_{j=1}^{v_{1}} \Gamma\left(\gamma_{j}-\xi\right) \prod_{j=1}^{m_{2}} \Gamma\left(\beta_{j}^{\prime}+\eta\right)}{\prod_{j=1}^{q_{1}} \Gamma\left(1-\beta_{j}-\xi\right) \prod_{j=v_{1}+1}^{t_{1}} \Gamma\left(1-\gamma_{j}+\xi\right) \prod_{j=m_{2}+1}^{q_{2}} \Gamma\left(1-\beta_{j}^{\prime}-\eta\right)} \\
& \times \frac{\prod_{j=1}^{v_{2}} \Gamma\left(\gamma_{j}^{\prime}-\eta\right) \prod_{j=1}^{n} \Gamma\left(1-\varepsilon_{j}-\xi-\eta\right)}{\prod_{j=v_{2}+1}^{t_{2}} \Gamma\left(1-\gamma_{j}^{\prime}+\eta\right) \prod_{j=n+1}^{p} \Gamma\left(\varepsilon_{j}+\xi+\eta\right) \prod_{j=1}^{s} \Gamma\left(\delta_{j}-\xi-\eta\right)} x^{-\xi} y^{-\eta} \mathrm{d} \xi \mathrm{d} \eta .
\end{aligned}
$$

$$
\begin{aligned}
M_{\gamma}(s) & =\int_{0}^{\infty} \int_{0}^{\infty} \exp \left(\frac{-s \gamma_{1} \gamma_{2}}{\gamma_{2}+c}\right) f_{\gamma_{1}}\left(\gamma_{1}\right) f_{\gamma_{2}}\left(\gamma_{2}\right) \mathrm{d} \gamma_{1} \mathrm{~d} \gamma_{2} \\
& =A_{1} \int_{0}^{\infty}\left\{\int_{0}^{\infty} \gamma_{1}^{m_{1}-1} \exp \left[-\left(\frac{m_{1}}{\bar{\gamma}_{1}}+\frac{s \gamma_{2}}{\gamma_{2}+c}\right) \gamma_{1}\right] \mathrm{d} \gamma_{1}\right\} f_{\gamma_{2}}\left(\gamma_{2}\right) \mathrm{d} \gamma_{2} \\
& =A_{1} A_{2} \Gamma\left(m_{1}\right) \int_{0}^{\infty}\left(\frac{m_{1}}{\bar{\gamma}_{1}}+\frac{s \gamma_{2}}{\gamma_{2}+c}\right)^{-m_{1}} \gamma_{2}^{m_{2}-1} \exp \left(-\frac{m_{2}}{\bar{\gamma}_{2}} \gamma_{2}\right) \mathrm{d} \gamma_{2} \\
& =A_{1} A_{2} \Gamma\left(m_{1}\right)\left(\frac{\bar{\gamma}_{1}}{m_{1}+s \bar{\gamma}_{1}}\right)^{m_{1}} \int_{0}^{\infty} \frac{\left(\gamma_{2}+c\right)^{m_{1}} \gamma_{2}^{m_{2}-1} \exp \left(-\frac{m_{2}}{\bar{\gamma}_{2}} \gamma_{2}\right)}{\left(\gamma_{2}+\frac{c m_{1}}{m_{1}+s \bar{\gamma}_{1}}\right)^{m_{1}} \mathrm{~d} \gamma_{2}} \\
& =A_{1} A_{2} \Gamma\left(m_{1}\right)\left(\frac{\bar{\gamma}_{1}}{m_{1}+s \bar{\gamma}_{1}}\right)^{m_{1}} \int_{0}^{\infty} \frac{\left(\gamma_{2}+c\right)^{\left\lceil m_{1}\right\rceil} \gamma_{2}^{m_{2}-1} \exp \left(-\frac{m_{2}}{\bar{\gamma}_{2}} \gamma_{2}\right)}{\left(\gamma_{2}+\frac{c m_{1}}{m_{1}+s \bar{\gamma}_{1}}\right)^{m_{1}}\left(\gamma_{2}+c\right)^{\left\lceil m_{1}\right\rceil-m_{1}}} \mathrm{~d} \gamma_{2} \\
& =\frac{A_{1} A_{2} \Gamma\left(m_{1}\right)}{m_{1} m_{1} \bar{\gamma}_{1}^{-m_{1}}} \sum_{k=0}^{\left\lceil m_{1}\right\rceil}\left(\begin{array}{c}
\left\lceil m_{1}\right\rceil \\
k
\end{array}\right)^{-k} \int_{0}^{\infty} \frac{\gamma_{2}^{k+m_{2}-1} \exp \left(-\frac{m_{2}}{\bar{\gamma}_{2}} \gamma_{2}\right)}{\left(1+\frac{m_{1}+s \bar{\gamma}_{1}}{c m_{1}} \gamma_{2}\right)^{m_{1}}\left(1+\frac{1}{c} \gamma_{2}\right)^{\left\lceil m_{1}\right\rceil-m_{1}}} \mathrm{~d} \gamma_{2} .
\end{aligned}
$$

$$
\begin{aligned}
& T \triangleq \int_{0}^{\infty} \frac{\gamma^{k+m_{2}-1} \exp \left(-\frac{m_{2} \gamma}{\bar{\gamma}_{2}}\right)}{\left(1+\frac{\left(m_{1}+s \bar{\gamma}_{1}\right) \gamma}{c m_{1}}\right)^{m_{1}}\left(1+\frac{\gamma}{c}\right)^{\left\lceil m_{1}\right\rceil-m_{1}}} \mathrm{~d} \gamma \\
& =C \int_{0}^{\infty} \gamma^{k+m_{2}-1} \exp \left(-\frac{m_{2} \gamma}{\bar{\gamma}_{2}}\right) \\
& \times H_{1,1}^{1,1}\left[\begin{array}{c|c}
\left(m_{1}+s \bar{\gamma}_{1}\right) \gamma \\
c m_{1} & \left(1-m_{1}, 1\right) \\
(0,1)
\end{array}\right] H_{1,1}^{1,1}\left[\frac{\gamma}{c} \mid \begin{array}{c}
\left(1-\left\lceil m_{1}\right\rceil+m_{1}, 1\right) \\
(0,1)
\end{array}\right] \mathrm{d} \gamma \\
& =C\left(\frac{\bar{\gamma}_{2}}{m_{2}}\right)^{k+m_{2}} H_{1,[1: 1], 0,[1: 1]}^{1,1,1,1}\left[\begin{array}{c|c}
\frac{\left(m_{1}+s \bar{\gamma}_{1}\right) \bar{\gamma}_{2}}{c m_{1} m_{2}} & \left(k+m_{2}, 1\right) \\
\frac{\bar{\gamma}_{2}}{c m_{2}} & \left(1-m_{1}, 1\right) ;\left(1-\left\lceil m_{1}\right\rceil+m_{1}, 1\right) \\
- & (0,1) ;(0,1)
\end{array}\right] \\
& =C\left(\frac{\bar{\gamma}_{2}}{m_{2}}\right)^{k+m_{2}} G_{1,[1: 1], 0,[1: 1]}^{1,1,1,1,1}\left[\begin{array}{c|c}
\frac{\left(m_{1}+s \bar{\gamma}_{1}\right) \bar{\gamma}_{2}}{c m_{1} m_{2}} & k+m_{2} \\
\frac{\bar{\gamma}_{2}}{c m_{2}} & 1-m_{1} ; 1-\left\lceil m_{1}\right\rceil+m_{1} \\
- & 0 ; 0
\end{array}\right] \text {. }
\end{aligned}
$$




$$
\begin{aligned}
& =\Gamma\left(a_{1}\right) \Gamma\left(1-b_{1}\right) \sum_{m=0}^{\infty} \sum_{n=0}^{\infty} \frac{\left(a_{1}\right)_{m+n}\left(1-b_{1}\right)_{m}(0)_{n}}{m ! n !} \\
& \times(-x)^{m}(-y)^{n} \\
& =\Gamma\left(a_{1}\right) \Gamma\left(1-b_{1}\right) \sum_{m=0}^{\infty} \frac{\left(a_{1}\right)_{m}\left(1-b_{1}\right)_{m}}{m !}(-x)^{m} \\
& =\Gamma\left(a_{1}\right) \Gamma\left(1-b_{1}\right){ }_{2} F_{0}\left(a_{1}, 1-b_{1} ;-;-x\right) \\
& =G_{1,2}^{2,1}\left(\frac{1}{x} \mid \begin{array}{c}
1 \\
a_{1}, 1-b_{1}
\end{array}\right),
\end{aligned}
$$

where [26, Eqs. (5. 6. (1)) \& (5.3.1. (9))] are exploited to reach (43), respectively. Notice that a typo in [26, Eq. (5.6. (1))] was pointed out in the errata.

\section{APPENDIX D \\ PRoOf OF LEMMA 1}

The $n^{\text {th }}$-order moment of the end-to-end SNR in (3) can be calculated as

$$
\begin{aligned}
\mathbb{E}\left\{\gamma^{n}\right\}= & \int_{0}^{\infty} \int_{0}^{\infty}\left(\frac{\gamma_{1} \gamma_{2}}{\gamma_{2}+c}\right)^{n} f_{\gamma_{1}}\left(\gamma_{1}\right) f_{\gamma_{2}}\left(\gamma_{2}\right) \mathrm{d} \gamma_{1} \mathrm{~d} \gamma_{2} \\
= & \int_{0}^{\infty} \gamma_{1}^{n} f_{\gamma_{1}}\left(\gamma_{1}\right) \mathrm{d} \gamma_{1} \int_{0}^{\infty}\left(\frac{\gamma_{2}}{\gamma_{2}+c}\right)^{n} f_{\gamma_{2}}\left(\gamma_{2}\right) \mathrm{d} \gamma_{2} \\
= & A_{1} A_{2} \int_{0}^{\infty} \gamma_{1}^{m_{1}+n-1} \exp \left(-\frac{m_{1}}{\bar{\gamma}_{1}} \gamma_{1}\right) \mathrm{d} \gamma_{1} \\
& \times \int_{0}^{\infty} \frac{\gamma_{2}^{m_{2}+n-1}}{\left(\gamma_{2}+c\right)^{n}} \exp \left(-\frac{m_{2}}{\bar{\gamma}_{2}} \gamma_{2}\right) \mathrm{d} \gamma_{2} \\
= & A_{1} A_{2} \Gamma\left(m_{1}+n\right) \Gamma\left(m_{2}+n\right) c^{m_{2}}\left(\frac{\bar{\gamma}_{1}}{m_{1}}\right)^{m_{1}+n} \\
& \times \Psi\left(m_{2}+n, m_{2}+1 ; \frac{c m_{2}}{\bar{\gamma}_{2}}\right),
\end{aligned}
$$

where we exploited [21, vol. 1, Eqs. (2.3.3.1)\& (2.3.6.9)] in (44). Finally, substituting $A_{1}$ and $A_{2}$ from (5) into (44) followed by some manipulations leads to (16).

\section{REFERENCES}

[1] J. N. Laneman, D. N. C. Tse, and G. W. Wornell, "Cooperative diversity in wireless networks: efficient protocols and outage behaviour," IEEE Trans. Inf. Theory, vol. 50, no. 12, pp. 3062-3080, Dec. 2004.

[2] M. O. Hasna and M.-S. Alouini, "A performance study of dual-hop transmission with fixed gain relays," IEEE Trans. Wireless Commun., vol. 3, no. 6, pp. 1963-1968, Nov. 2004.

[3] Y. Li and S. Kishore, "Asymptotic analysis of amplify-and-forward relaying in Nakagami-fading environments," IEEE Trans. Wireless Commun., vol. 6, no. 12, pp. 4256-4262, Nov. 2007.

[4] Y. A. Chau and K. Y. Huang, "Channel statistics and performance of cooperative selection diversity with dual-hop amplify-and-forward relay over Rayleigh fading channels," IEEE Trans. Wireless Commun., vol. 7, no. 5, pp. 1779-1785, May 2008.

[5] D. B. da Costa and S. Aïssa, "End-to-end performance of dual-hop semi-blind relaying systems with partial relay selection," IEEE Trans. Wireless Commun., vol. 8, no. 8, pp. 4306-4315, Aug. 2009.

[6] X. J. Zhang, Y. Gong, and K. B. Letaief, "On the diversity gain in cooperative relaying channels with imperfect CSIT," IEEE Trans. Commun., vol. 58, no. 4, pp. 1273-1279, Apr. 2010.

[7] S. S. Ikki and S. Aïssa, "Performance analysis of dual-hop relaying systems in the presence of co-channel interference," in Proc. IEEE Global Telecom. Conf., Dec. 2010, pp. 1-5.

[8] T. A. Tsiftsis, G. K. Karagiannidis, P. T. Mathiopoulos, and S. A. Kotsopoulos, "Nonregenerative dual-hop cooperative links with selection diversity," EURASIP J. Wireless Commun. and Networking, vol. 2006, pp. 1-8, May 2006.
[9] D. B. da Costa and S. Aïssa, "Capacity analysis of cooperative systems with relay selection in Nakagami- $m$ fading," IEEE Commun. Lett., vol. 13, no. 9, pp. 637-639, Sep. 2009.

[10] H. A. Suraweera and G. K. Karagiannidis, "Closed-form error analysis of the non-identical Nakagami- $m$ relay fading channel," IEEE Commun. Lett., vol. 12, no. 4, pp. 259-261, Apr. 2008.

[11] S. S. Ikki and M. H. Ahmed, "Performance of cooperative diversity using equal gain combining (EGC) over Nakagami- $m$ fading channels," IEEE Trans. Wireless Commun., vol. 8, no. 2, pp. 557-562, Feb. 2009.

[12] D. B. da Costa and S. Aïssa, "Cooperative dual-hop relaying systems with beamforming over Nakagami- $m$ Fading channels," IEEE Trans. Wireless Commun., vol. 8, no. 8, pp. 3950-3954, Aug. 2009.

[13] S. Alapattu, N. Rajatheva, and C. Tellambura, "Performance analysis of TDMA relay protocols over Nakagami-m fading," IEEE Trans. Veh. Technol., vol. 59, no. 1, pp. 93-104, Jan. 2010.

[14] N. Yang, M. Elkashlan, and J. Yuan, "Outage probability of multiuser relay networks in Nakagami- $m$ fading channels," IEEE Trans. Veh. Technol., vol. 59, no. 5, pp. 2120-2132, June 2010.

[15] M. D. Yacoub, "The $\kappa-\mu$ distribution and the $\eta-\mu$ distribution," IEEE Antennas Propag. Mag., vol. 49, no. 1, pp. 68-81, Feb. 2007.

[16] L. Rubio, J. Reig, and N. Cardona, "Evaluation of Nakagami fading behaviour based on measurements in urban scenarios," Elsevier Int. J. Electron. Commun., vol. 61, pp. 135-138, 2007.

[17] L. L. Yang and H.-H. Chen, "Error probability of digital communications using relay diversity over Nakagami- $m$ fading channels," IEEE Trans. Wireless Commun., vol. 7, no. 5, pp. 1806-1811, May 2008.

[18] H. Shin and J. B. Song, "MRC analysis of cooperative diversity with fixed-gain relays in Nakagami- $m$ fading channels," IEEE Trans. Wireless Commun., vol. 7, no. 6, pp. 2069-2074, June 2008.

[19] M. D. Renzo, F. Graziosi, and F. Santucci, "A comprehensive framework for performance analysis of dual-hop cooperative wireless systems with fixed-gain relays over generalized fading channels," IEEE Trans. Wireless Commun., vol. 8, no. 10, pp. 5060-5074, Oct. 2009.

[20] M. K. Simon and M.-S. Alouini, Digital Communication over Fading Channels, 2nd edition. John Weley \& Sons Inc., 2005.

[21] A. P. Prudnikov, Y. A. Brychkov, and O. I. Marichev, Integrals and Series. Gordon and Breach Science Publishers, 1986.

[22] A. M. Mathai and R. K. Saxena, "Expansion of Meijer's $G$-function of two variables when the upper parameters differ by integers," Kyungpook Math. J., vol. 12, no. 1, pp. 61-68, June 1972.

[23] R. P. Agarwal, "An extension of Meijer's G-function," Proc. Nat. Inst. Sci. India, part A, vol. 31, pp. 536-546, 1965.

[24] A. M. Mathai and R. K. Saxena, The H-function with Applications in Statistics and Other Disciplines. Wiley Eastern, 1978.

[25] I. S. Ansari, S. Al-Ahmadi, F. Yilmaz, M.-S. Alouini, and H. Yanikomeroglu, "A new formula for the BER of binary modulations with dual-brance selection over gereralized- $K$ composite fading channels," preprint. Available: http://arxiv.org/abs/1012.3788.

[26] A. Erdélyi, Higher Transcendental Functions, vol. 1. McGraw-Hill Book Company, Inc., 1953.

[27] J. L. Burchnall and T. W. Chaundy, "Expansion of Appell's double hypergeometric functions," Quarterly J. Mathematics, vol. 11, pp. 249$270,1940$.

[28] M. Abramowitz and I. A. Stegun, Handbook of Mathematical Functions with Formulas, Graphs, and Mathematical Tables. Dover, 1972.

[29] A. Maaref and S. Aissa, "Exact error probability analysis of rectangular QAM for single- and multichannel reception in Nakagami- $m$ fading channels," IEEE Trans. Commun., vol. 57, no. 1, pp. 214-221, Jan. 2009.

[30] V. Asghari, A. Maaref, and S. Aïssa, "Symbol error probability analysis for multihop relaying over Nakagami fading channels," in Proc. IEEE Wireless Communications and Networking Conference, Apr. 2010.

[31] 3GPP, "Evolved universal terrestrial radio access: physical channels and modulation," TS 36.211, v9.0.0, Dec. 2009. Available: http://www.3gpp.org/FTP/Specs/html-info/36211.htm.

[32] M. R. McKay, A. Zanella, I. B. Collings, and M. Chiani, "Error probability and SINR analysis of optimum combining in Rician fading," IEEE Trans. Commun., vol. 57, no. 3, pp. 676-687, Mar. 2009.

[33] M. Di Renzo, F. Graziosi, and F. Santucci, "Channel capacity over generalized fading channels: a novel MGF-based approach for performance analysis and design of wireless communication systems," IEEE Trans. Veh. Technol., vol. 59, no. 1, pp. 127-149, Jan. 2010.

[34] Z. Wang and G. B. Giannakis, "A simple and general parameterization quantifying performance in fading channels," IEEE Trans. Commun., vol. 51, no. 8, pp. 1389-1398, Aug. 2003.

[35] L. C. Andrews, Special Functions of Mathematics for Engineers., 2nd edition. McGraw-Hill, Inc., 1992. 


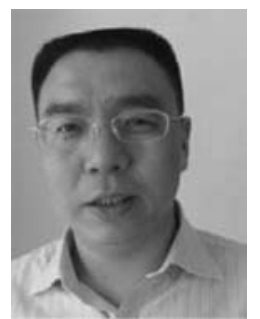

Minghua Xia obtained his Ph.D. degree in Telecommunications and Information Systems from Sun Yatsen University, Guangzhou, China, in 2007. From March 2007 to July 2009, he was with the Electronics and Telecommunications Research Institute (ETRI) of South Korea, Beijing R\&D Center, Beijing, China, where he worked as a member of engineering staff and participated in the projects on the physical layer design of 3GPP LTE mobile communications. From Aug. 2009 to Feb. 2011, he was with The University of Hong Kong (HKU), Hong Kong, as a Postdoctoral Fellow. Currently, he is with King Abdullah University of Science and Technology (KAUST), Saudi Arabia. His research interests are in the area of network information theory and space-time signal processing, and in particular the design and performance analysis of multiuser multi-antenna systems, cooperative relaying systems and cognitive radio networks.

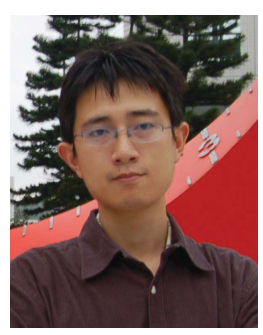

Chengwen Xing received the B.Eng. degree from Xidian University, Xi'an, China, in 2005 and the Ph.D. degree in the Department of Electrical and Electronic Engineering, The University of Hong Kong, Hong Kong, in 2010. Since September 2010, he has been with the School of Information and Electronics, Beijing Institute of Technology (BIT), Beijing, China, where he is currently a Lecturer. He was a TPC member for ICC 2011 and Globecom 2011. His current research interests include statistical signal processing, convex optimization, multivariate statistics, combinatorial optimization and cooperative communication systems.

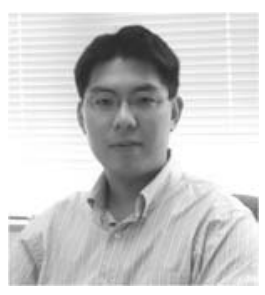

Yik-Chung Wu obtained the B.Eng. (EEE) degree in 1998 and the M.Phil. degree in 2001 from The University of Hong Kong (HKU), and Ph.D. degree in 2005 from Texas A\&M University, USA. From Aug. 2005 to Aug. 2006, he was with the Thomson Corporate Research, Princeton, NJ, as a Member of Technical Staff. Since Sep. 2006, he has been with The University of Hong Kong as an Assistant Professor. Yik-Chung's research interests are in general area of signal processing and communication systems, and in particular receiver algorithm design, synchronization techniques, channel estimation and equalization. He is currently serving as an associate editor for the IEEE COMMUNICATIONS LETTERS.

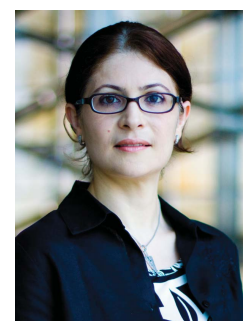

Sonia Aïssa (S'93-M'00-SM'03) received her Ph.D. degree in Electrical and Computer Engineering from McGill University, Montreal, QC, Canada, in 1998. Since then, she has been with the National Institute of Scientific Research-Energy, Materials, and Telecommunications (INRS-EMT), University of Quebec, Montreal, QC, Canada, where she is a Professor.

From 1996 to 1997, she was a Researcher with the Department of Electronics and Communications of Kyoto University, Kyoto, Japan, and with the Wireless Systems Laboratories of NTT, Kanagawa, Japan. From 1998 to 2000, she was a Research Associate at INRS-EMT, Montreal. From 2000 to 2002, while she was an Assistant Professor, she was a Principal Investigator in the major program of personal and mobile communications of the Canadian Institute for Telecommunications Research (CITR), leading research in radio resource management for code division multiple access systems. From 2004 to 2007, she was an Adjunct Professor with Concordia University, Montreal. In 2006, she was Visiting Invited Professor with the Graduate School of Informatics, Kyoto University, Kyoto, Japan. Her research interests lie in the area of wireless and mobile communications, and include radio resource management, cross-layer design and optimization, design and analysis of multiple antenna (MIMO) systems, cognitive and cooperative transmission techniques, and performance evaluation, with a focus on Cellular, Ad Hoc, and Cognitive Radio networks.

Dr. Aïssa was the Founding Chair of the Montreal Chapter IEEE Women in Engineering Society in 2004-2007, acted or is currently acting as Technical Program Leading Chair or Cochair for the Wireless Communications Symposium of the IEEE International Conference on Communications (ICC) in 2006, 2009, 2011 and 2012, as PHY/MAC Program Chair for the 2007 IEEE Wireless Communications and Networking Conference (WCNC), and as Technical Program Committee Cochair of the 2013 IEEE Vehicular Technology Conference - spring (VTC). She has served as a Guest Editor of the EURASIP Journal on Wireless Communications and Networking in 2006, and as Associate Editor of the IEEE Wireless Communications Magazine in 20062010. She is currently an Editor of the IEEE TRANSACTIONS ON WIRELESS COMMUNICATIONS, the IEEE TRANSACTIONS ON COMMUNICATIONS and the IEEE Communications Magazine, and Associate Editor of the Wiley Security and Communication Networks Journal. Awards and distinctions to her credit include the Quebec Government FQRNT Strategic Fellowship for Professors-Researchers in 2001-2006; the INRS-EMT Performance Award in 2004 for outstanding achievements in research, teaching and service; the IEEE Communications Society Certificate of Appreciation in 2006-2011; and the Technical Community Service Award from the FQRNT Center for Advanced Systems and Technologies in Communications (SYTACom) in 2007. She is also co-recipient of Best Paper Awards from IEEE ISCC 2009, WPMC 2010, IEEE WCNC 2010 and IEEE ICCIT 2011; and recipient of NSERC (Natural Sciences and Engineering Research Council of Canada) Discovery Accelerator Supplement Award. 